\title{
Stability of Projection Methods for Incompressible Flows Using High Order Pressure-Velocity Pairs of Same Degree: Continuous and Discontinuous Galerkin Formulations
}

\author{
E. Ferrer ${ }^{1, *}$, D. Moxey ${ }^{2}$, R. H. J. Willden ${ }^{3}$ and S. J. Sherwin ${ }^{2}$ \\ ${ }^{1}$ ETSIA - School of Aeronautics, Universidad Politécnica de Madrid, Pza. Cardenal \\ Cisneros 3, Madrid, E-28040, Spain. \\ 2 Department of Aeronautics, Imperial College London, London, SW7 2AZ, UK. \\ ${ }^{3}$ Department of Engineering Science, University of Oxford, Parks Road, Oxford, \\ OX1 3PJ, UK.
}

Received 29 January 2014; Accepted (in revised version) 17 April 2014

Available online 17 July 2014

\begin{abstract}
This paper presents limits for stability of projection type schemes when using high order pressure-velocity pairs of same degree. Two high order $h / p$ variational methods encompassing continuous and discontinuous Galerkin formulations are used to explain previously observed lower limits on the time step for projection type schemes to be stable [18], when $h$ - or $p$-refinement strategies are considered. In addition, the analysis included in this work shows that these stability limits do not depend only on the time step but on the product of the latter and the kinematic viscosity, which is of particular importance in the study of high Reynolds number flows. We show that high order methods prove advantageous in stabilising the simulations when small time steps and low kinematic viscosities are used.

Drawing upon this analysis, we demonstrate how the effects of this instability can be reduced in the discontinuous scheme by introducing a stabilisation term into the global system. Finally, we show that these lower limits are compatible with CourantFriedrichs-Lewy (CFL) type restrictions, given that a sufficiently high polynomial order or a mall enough mesh spacing is selected.
\end{abstract}

AMS subject classifications: 76D05, 35Q30, 65N30

Key words: Incompressible Navier-Stokes equations, projection methods, velocity-correction, continuous Galerkin, discontinuous Galerkin, inf-sup LBB condition.

*Corresponding author. Email addresses: esteban.ferrer@upm.es (E. Ferrer), d.moxey@imperial.ac.uk (D. Moxey), richard.willden@eng.ox.ac.uk (R. H. J. Willden), s.sherwin@imperial.ac.uk (S. J. Sherwin) 


\section{Introduction}

Since the introduction of projection type algorithms for the efficient solution of the incompressible Navier-Stokes equations in primitive variables by Chorin [8] and Temam [35], various modifications have emerged. These variations, whereby intermediate variables enable some degree of decoupling of pressure and velocity, may be viewed as one of three categories: pressure-correction, velocity projection and consistent splitting methods [18]. These methods share the appealing property of requiring the solution of decoupled elliptic equations for the velocity and pressure fields, which renders these type of schemes very efficient and thus extremely useful in numerical simulations.

For many years, it had been thought that this category of schemes do not require an inf-sup condition to be fulfilled since velocity and pressure are essentially "decoupled". The inf-sup (or LBB from Ladyzhenskaya [26], Babushka [3] and Brezzi [5]) condition typically states that an equal order expansion for both pressure and velocity leads to an unstable system $[6,13,23]$. To overcome this difficulty one can augment the velocity space with respect to the pressure space or add stabilisation whilst maintaining the same space dimensions for pressure and velocity.

Projection-type schemes have historically been implemented using equal order spaces for pressure and velocity since they appeared to be stable in this setting. However, in recent years it has been shown that these schemes instead correspond to stabilised-like schemes [4,10,18]. Auteri et al. [2] and Guermond et al. [18] provided a summary of the stability conditions for different projection schemes by reducing the analysis of the unsteady incompressible Navier-Stokes equations to the equivalent steady Stokes problem. This same approach will be considered in following sections. A particularly interesting conclusion from [18] is that the stability condition for the Chorin-Temam scheme to be stable depends on the time step $\triangle t$. Namely, the time step is required to be large enough for the scheme to remain stable. Observations are reported for the time step limit for stability $\triangle t_{\text {lim }}$ when considering low order (e.g. linear finite element formulations, relying only on $h$-refinement) and high order spectral type methods (e.g. Fourier, Chebyshev, allowing $p$-refinement):

- $\triangle t_{\text {lim }} \geq c_{1} h^{2}$ for low order finite element methods, where $c_{1}$ is a constant independent of the spatial discretisation defined through the characteristic mesh size $h$,

- $\triangle t_{\text {lim }} \geq c_{2} k^{-3}$ for high order spectral discretisations, where $c_{2}$ is a constant independent of the spatial discretisation defined through the polynomial order $k$.

In this work, we examine these observations and provide an explanation for such behaviour. Without loss of generality, we focus on the analysis of the popular velocitycorrection scheme proposed by Orszag et al. [29], Karniadakis et al. [24] and theoretically analysed by Guermond et al. [18], which under the steady Stokes assumption can be shown to resemble the Chorin-Temam scheme. In particular, we will study the effects of using such projection schemes together with high order spatial discretisations, 
enabling both $h$ - and $p$-type refinement, which to the authors' knowledge has not been fully addressed in the past. To this end, the results presented in this work rely on twodimensional computations obtained using a high order $h / p$ continuous Galerkin (CG) [25] and a discontinuous Galerkin (DG) solver [15-17]. Both CG and DG methods allow $h$ - and $p$-refinement strategies to be performed independently. This characteristic enables the analysis of the effect of both types of refinement independently on the stability of the temporal scheme. The use of both schemes additionally allows us to examine stability properties under different spatial discretisations.

We summarise in the following the main contributions of this work:

- We confirm through examples and analysis that projection schemes such as the velocity-correction scheme proposed by Orszag et al. [29] and Karniadakis et al. [24] correspond to stabilised-like schemes.

- We outline the dependency of the time step limit for stability $\triangle t_{\text {lim }}$ (as observed in [18] and references therein) with respect to the spatial discretisation using either $h$ or $p$ refinement.

- We analytically derive estimates for the limits of stability observed previously: $\triangle t_{\text {lim }} \geq c_{1} h^{2}$ for low order finite element methods, and $\triangle t_{\text {lim }} \geq c_{2} k^{-3}$ for high order spectral discretisations.

- We show that the limit for stability depends not only on the time step, but the parameter $\kappa=v \triangle t$ where $v$ is the kinematic viscosity. We note that this is particularly relevant for simulations of industrial interest, where the Reynolds number is typically high, leading to a small kinematic viscosity.

- We show that high order spatial discretisations (i.e. high polynomial orders) prove advantageous to relax the limit for stability $\kappa_{\lim }$, enabling reduced values for the time step and/or the kinematic viscosity.

- We show that the limit for $\kappa_{\lim }$ is compatible with the Courant-Friedrichs-Lewy (CFL) restriction if high order spatial discretisations are considered together with an explicit treatment of the non-linear terms (i.e. full Navier-Stokes equations).

- Finally, we propose a possible stabilisation technique for low order spatial discretisation, which takes advantage of the interior penalty discontinuous Galerkin formulation used throughout this work. This technique is utilised to confirm the previous analysis.

Additionally, we note that very recently [34] reported a similar type of instability as presented in this work, which was attributed to the use of a non-conformal DG formulation. However, in the present work we show that these instabilities are inherent to the temporal scheme and independent of the spatial discretisation (and the continuity/discotninuity of the underlying spaces).

The organisation of this paper is as follows. We begin in Section 2 by outlining the velocity-correction scheme together with the continuous and discontinuous Galerkin 
spatial discretisations, where it proves advantageous to write the discretised equations in their algebraic form. In Section 3 we consider the simulation of an unsteady Stokes problem to illustrate the stability properties of the scheme with respect to both the time step and the kinematic viscosity. From this, in Section 4 the analysis of the discretised system provides a relationship between spatial and temporal discretisations for the steady Stokes problem, showing that high order spatial discretisations or small mesh resolutions are required to compensate for small time steps or small kinematic viscosities and demonstrate stability depends on the parameter $\kappa=v \Delta t$. We also outline a possible low order stabilisation strategy for the DG formulation. Finally, in Section 5 we show that the inf-sup limit for stability $\kappa_{\lim }$ does not conflict with the time step required to fulfill the CFL condition derived from the full Navier-Stokes equations, as long as the polynomial order selected is sufficiently large or the mesh size is small enough.

\section{Methodology}

In what follows, we consider the two-dimensional incompressible Navier-Stokes (NS) equations. Let $\Omega$ be a domain in $\mathbb{R}^{2}$ with boundary $\partial \Omega=\partial \Omega_{D} \cup \partial \Omega_{N}$ composed of Dirichlet $\left(\partial \Omega_{D}\right)$ or Neumann $\left(\partial \Omega_{N}\right)$ parts, where $\partial \Omega_{D} \cap \partial \Omega_{N}=\varnothing$. We denote the outward unit normal vector to $\partial \Omega$ by $\mathbf{n}$. The unsteady dimensional incompressible NS equations read:

$$
\begin{array}{ll}
\frac{\partial \mathbf{u}}{\partial t}+\mathbf{N}(\mathbf{u})=-\nabla p+v \nabla^{2} \mathbf{u} & \text { in } \Omega \times[0, T], \\
\nabla \cdot \mathbf{u}=0 & \text { in } \Omega \times[0, T], \\
\mathbf{u}(t=0)=\mathbf{u}_{\mathbf{0}} & \text { in } \Omega, \\
\mathbf{u}=\mathbf{L}_{\mathbf{D}} & \text { on } \partial \Omega_{D} \times[0, T], \\
\nu \frac{\partial \mathbf{u}}{\partial n}-p \mathbf{n}=\mathbf{0} & \text { on } \partial \Omega_{N} \times[0, T],
\end{array}
$$

where $t$ represents time, $\mathbf{u}=(u, v)^{\top}$ the velocity vector, $p$ the static pressure to density ratio and $v$ the kinematic viscosity. In addition, $\mathbf{u}_{0}$ represents an initial condition for the velocity field and $\mathbf{L}_{\mathbf{D}}$ defines the Dirichlet boundary data for the velocity. $\mathbf{N}(\mathbf{u})$ represents the non-linear contributions expressed, for example, in convective form $\mathbf{N}(\mathbf{u})=(\mathbf{u} \cdot \nabla) \mathbf{u}$. For a discussion on different non-linear term formulations, the reader is referred to [25] and references therein.

\subsection{Temporal discretisation of the incompressible NS equations}

There is a vast amount of literature on projection type procedures for the solution of the incompressible NS equations and reviews can be found in $[11,18,25]$. In this work, we select a second order velocity-correction (sometimes referred to as dual stiffly stable method) developed by Orszag et al. [29] and Karniadakis et al. [24] to discretise the equations in time. The NS equations are integrated in time using a stiffly-stable scheme with 
coefficients $\gamma_{0}, \alpha_{n}$ and $\beta_{n}$. The resulting equation is separated using two intermediate variables $\tilde{\mathbf{u}}$ and $\tilde{\mathbf{u}}$ into three steps: an explicit non-linear advection, an implicit pressure solve and an implicit velocity correction, resulting in the scheme

$$
\begin{aligned}
& \frac{\gamma_{0} \tilde{\mathbf{u}}-\sum_{q=0}^{J-1} \alpha_{q} \mathbf{u}^{n-q}}{\Delta t}=-\sum_{q=0}^{J-1} \beta_{q} \mathbf{N}\left(\mathbf{u}^{n-q}\right), \\
& \gamma_{0} \frac{\tilde{\tilde{\mathbf{u}}}-\tilde{\mathbf{u}}}{\Delta t}=-\nabla p^{n+1}, \\
& \gamma_{0} \frac{\mathbf{u}^{n+1}-\tilde{\mathbf{u}}}{\Delta t}=v \nabla^{2} \mathbf{u}^{n+1} .
\end{aligned}
$$

Here $\mathbf{u}^{n}$ denotes $\mathbf{u}$ evaluated at time $t=n \Delta t$, for $n \in \mathbb{N}$. The temporal accuracy is determined by the parameter $J$, which we fix at two for this work (i.e. $\gamma_{0}=3 / 2, \alpha_{0}=2$, $\alpha_{1}=-1 / 2, \beta_{0}=2$ and $\beta_{1}=-1$ ). Note that in order to solve for the pressure field we assume that the second intermediate velocity field is solenoidal so that $\nabla \cdot \tilde{\tilde{\mathbf{u}}}=0$, and take the divergence of Eq. (2.2) to obtain the pressure Poisson equation

$$
-\nabla^{2} p^{n+1}=-\frac{\gamma_{0}}{\Delta t} \nabla \cdot \tilde{\mathbf{u}}
$$

This equation must be equipped with a suitable boundary condition where solid walls are present to avoid numerical errors which would otherwise affect the temporal accuracy of the scheme [24]. This takes the form of a Neumann condition

$$
{\frac{\partial p^{n+1}}{\partial n}}^{n}=-\mathbf{n} \cdot{\frac{\partial \mathbf{u}^{n+1}}{\partial t}}^{J-n} \cdot \sum_{q=0}^{J-1} \beta_{q}\left(\mathbf{N}\left(\mathbf{u}^{n-q}\right)+v \nabla \times \boldsymbol{\omega}^{n-q}\right),
$$

where $\omega=\nabla \times \mathbf{u}$ is the vorticity. This expression indirectly enforces the incompressibility constraint and gives a consistent method. As noted in [18], this Neumann vorticity boundary condition requires the velocity to have computable second derivatives. For this reason, all computations are performed using polynomial orders $k \geq 2$.

\subsection{Spatial discretisations: continuous and discontinuous Galerkin methods}

We now briefly outline the spatial discretisation of the domain $\Omega \subset \mathbb{R}^{2}$. We begin by taking a conformal triangular tessellation of $N_{\mathrm{el}}$ elements $\Omega_{h}=\bigcup_{e=1}^{N_{\mathrm{el}}} \Omega^{e}$ which approximates $\Omega$. Interior edges (i.e. edges common to two elements) are denoted by $\Gamma_{h}$, and we partition the exterior boundary $\partial \Omega_{h}$ as $\Gamma_{D} \cup \Gamma_{N}$, where $\Gamma_{D} \cap \Gamma_{N}=\varnothing$ and $\Gamma_{D}$ and $\Gamma_{N}$ represent Dirichlet and Neumann boundaries respectively. For each triangle $\Omega^{e}$ with boundary $\partial \Omega^{e}$, we denote $\Gamma_{n}^{e}$ as the positively oriented (i.e. anticlockwise) single edge with $n=1,2,3$ and its associated outwards pointing normal vector as $\mathbf{n}_{\Gamma_{n}^{e}}$.

The aim of these discretisations is to calculate an approximate solution $u_{h}$ to the elliptic equations (2.2) and (2.3). We therefore consider the general Helmholtz formulation 


$$
\begin{array}{ll}
-\triangle u+\alpha u=f & \text { in } \Omega, \\
u=L_{D} & \text { on } \Gamma_{D}, \\
\nabla u \cdot \mathbf{n}=L_{N} & \text { on } \Gamma_{N},
\end{array}
$$

where $u \in H^{1}(\Omega)$ is a scalar solution (but extension to the vector formulation is direct), $\alpha \geq 0$ represents the wave number for the Helmholtz equation, $f \in L^{2}(\Omega)$ is the forcing term, and $L_{D} \in H^{1 / 2}\left(\Gamma_{D}\right)$ and $L_{N} \in L^{2}\left(\Gamma_{N}\right)$ represent Dirichlet and Neumann boundary data respectively.

\subsubsection{Expansion functions}

In either the continuous or discontinuous formulation, we first represent the approximate solution $u_{h}$ by an expansion in terms of basis functions

$$
u_{h}(\mathbf{x})=\sum_{i=0}^{N_{\mathrm{dof}}} \hat{u}_{i} \Phi_{i}(\mathbf{x})
$$

where the global modes $\Phi_{i} \in D_{k}\left(\Omega_{h}\right)$ are selected from spaces containing either continuous or discontinuous functions,

$$
\begin{aligned}
& D_{k}^{\mathrm{CG}}\left(\Omega_{h}\right)=\left\{v_{h} \in C^{0}\left(\Omega_{h}\right):\left.v_{h}\right|_{\Omega^{e}} \in \mathbb{P}_{k}\left(\Omega^{e}\right)\right\}, \\
& D_{k}^{\mathrm{DG}}\left(\Omega_{h}\right)=\left\{v_{h} \in L^{2}\left(\Omega_{h}\right):\left.v_{h}\right|_{\Omega^{e}} \in \mathbb{P}_{k}^{\prime}\left(\Omega^{e}\right)\right\},
\end{aligned}
$$

where $\mathbb{P}_{k}, \mathbb{P}_{k}^{\prime}$ denote spaces of polynomials of order less than or equal to $k$. We note that, in the continuous case, the definition of this space is incompatible with the Dirichlet condition of Eq. (2.6). However in the following sections we will consider the imposition of Dirichlet boundary conditions in a purely weak fashion.

Instead of explicitly selecting the global expansion functions from these spaces, for each polynomial order $k$ we consider a local expansion on each element $\Omega^{e}$ by a tensor product of one-dimensional basis functions in the computational space $\left(\xi_{1}, \xi_{2}\right)$, so that

$$
\left.u_{h}^{l}(\mathbf{x})\right|_{\Omega^{e}}=\sum_{i=0}^{N} \hat{u}_{i}^{e} \varphi_{i}(\mathbf{x})=\sum_{p=0}^{k} \sum_{q=0}^{k-p} \hat{u}_{p q}^{e} \phi_{p}^{a}\left(\xi_{1}\right) \phi_{p q}^{b}\left(\xi_{2}\right),
$$

where $N=(k+1)(k+2) / 2$ is the number of local degrees of freedom on each triangle. The mapping between computational space $\left(\xi_{1}, \xi_{2}\right)$ and physical space $\left(x_{1}, x_{2}\right)$ is accomplished through a collapsed coordinate system (or Duffy coordinates) defined in $[12,25]$ with both CG and DG formulations.

In this work, we select modal hierarchical expansion functions, but note that other expansions (e.g. nodal or Lagrange polynomial based) are possible and provide similar results. In the DG formulation we do not require continuity across element boundaries 
which provides flexibility in the selection of appropriate basis functions. We therefore choose a hierarchical $L^{2}$-orthonormal expansion basis from [25] defined in computational space as:

$$
\begin{aligned}
\phi_{p}^{a}\left(\xi_{1}\right) & =\sqrt{\frac{2 p+1}{2}} P_{p}^{(0,0)}\left(\xi_{1}\right), \\
\phi_{p q}^{b}\left(\xi_{2}\right) & =\sqrt{p+q+1}\left(\frac{1-\xi_{2}}{2}\right)^{p} P_{q}^{(2 p+1,0)}\left(\xi_{2}\right),
\end{aligned}
$$

and $P_{p}^{(\alpha, \beta)}(z)$ is the $p$-th order Jacobi polynomial, with $\alpha, \beta>-1$ given constants.

In the $C^{0}$ continuous Galerkin expansion, we utilise a similar hierarchical modal scheme, which is augmented with linear modes in order to decouple interior and boundary degrees of freedom. These boundary modes enable the imposition of $C^{0}$ continuity between adjacent elements

$$
\begin{aligned}
& \phi_{p}^{a}\left(\xi_{1}\right)= \begin{array}{ll}
\frac{1-\xi_{1}}{2}, & p=0, \\
\frac{1-\xi_{1}}{2} \frac{1+\xi_{1}}{2} P_{p-1}^{(1,1)}\left(\xi_{1}\right), & 1 \leq p \leq k-1, \\
\frac{1+\xi_{1}}{2}, & p=k,
\end{array} \\
& \phi_{p q}^{b}\left(\xi_{2}\right)= \begin{cases}\phi_{q}^{a}\left(\xi_{2}\right), & i=0,0 \leq j \leq k, \\
\left(\frac{1-\xi_{2}}{2}\right)^{p+1}, & 1 \leq p \leq k-1, q=0, \\
\left(\frac{1-\xi_{2}}{2}\right)^{p+1} \frac{1+\xi_{2}}{2} P_{q-1}^{(2 p+1,1)}\left(\xi_{2}\right), & 1 \leq p \leq k-1,1 \leq q \leq k-1, \\
\phi_{q}^{a}\left(\xi_{2}\right), & p=k, 0 \leq j \leq k .\end{cases}
\end{aligned}
$$

For both discretisations, the vector of local coefficients $\hat{\mathbf{u}}^{l}=\left(\hat{\mathbf{u}}^{1}, \cdots, \hat{\mathbf{u}}^{N_{\mathrm{el}}}\right)^{\top}$, where $\hat{\mathbf{u}}^{e}$ denotes the local coefficients $\hat{u}_{i}^{e}$ as defined in equation (2.9), must be related to the global coefficients $\hat{\mathbf{u}}=\left(\hat{u}_{1}, \cdots, \hat{u}_{N_{\text {dof }}}\right)^{\top}$. In the discontinuous framework, since all elements are decoupled we have that $\hat{\mathbf{u}}^{l}=\hat{\mathbf{u}}$ (i.e. double valued degrees of freedom at element edges). In order to guarantee the continuity of $u_{h}$ in the $C^{0}$ formulation however, we must ensure that coefficients along each edge are equal wherever they meet at a common edge or vertex. To this end we define an assembly matrix $\mathcal{A}$ whereby $\hat{\mathbf{u}}=\mathcal{A}^{\top} \hat{\mathbf{u}}^{l}$ which sums contributions from connected degrees of freedom, ensuring that global coefficients are uniquely defined.

\subsubsection{Variational formulations}

To find the discrete weak solution associated with the continuous problem represented by Eq. (2.6), we construct bilinear and linear forms $a(\cdot, \cdot)$ and $\ell(\cdot)$ associated with the finite 
element discrete variational formulation arising from the weak form of the continuous problem. We therefore seek a solution $u_{h} \in D_{k}\left(\Omega_{h}\right)$ such that for all $v_{h} \in D_{k}\left(\Omega_{h}\right)$,

$$
a\left(u_{h}, v_{h}\right)=\ell\left(v_{h}\right) .
$$

In the $C^{0}$ formulation, we seek $u_{h} \in D_{k}^{C G}\left(\Omega_{h}\right)$ and derive these forms in the standard fashion by selecting test functions $v_{h} \in D_{k}^{C G}\left(\Omega_{h}\right)$ and integrating Eq. (2.6) by parts so that

$$
\begin{aligned}
& a^{\mathrm{CG}}\left(u_{h}, v_{h}\right)=\int_{\Omega_{h}} \nabla u_{h} \cdot \nabla v_{h} d x+\alpha \int_{\Omega_{h}} u_{h} v_{h} d x, \quad \forall v_{h} \in D_{k}^{\mathrm{CG}}\left(\Omega_{h}\right), \\
& \ell^{\mathrm{CG}}\left(v_{h}\right)=\int_{\Omega_{h}} v_{h} f d x+\int_{\Gamma_{N}} v_{h} L_{N} d s, \quad \forall v_{h} \in D_{k}^{\mathrm{CG}}\left(\Omega_{h}\right) .
\end{aligned}
$$

For the discontinuous formulation, we utilise an interior penalty method (see [1] for a review of the various DG methods available for elliptic problems) and in particular, the Symmetric Interior Penalty Galerkin (SIPG) method is retained for the spatial discretisation. The details of the derivation are omitted here but can be found in various references $[1,7,21]$. In this formulation we seek $u_{h} \in D_{k}^{\mathrm{DG}}\left(\Omega_{h}\right)$ and arrive at the bilinear form

$$
\begin{aligned}
a^{\mathrm{DG}}\left(u_{h}, v_{h}\right)=\sum_{e=1}^{N_{\mathrm{el}}} & {\left[\int_{\Omega^{e}} \nabla u_{h} \cdot \nabla v_{h} d x+\int_{\Omega^{e}} \alpha u_{h} v_{h} d x\right] } \\
& -\sum_{\Gamma \in \Gamma_{h} \cup \Gamma_{D}}\left[\int_{\Gamma}\left\{\left\{\nabla u_{h}\right\}\right\} \cdot \mathbf{n}_{\Gamma}\left[\left[v_{h}\right]\right] d s+\int_{\Gamma}\left\{\left\{\nabla v_{h}\right\}\right\} \cdot \mathbf{n}_{\Gamma}\left[\left[u_{h}\right]\right] d s\right] \\
& +\sum_{\Gamma \in \Gamma_{h} \cup \Gamma_{D}} \frac{\sigma}{|\Gamma|^{\beta}} \int_{\Gamma}\left[\left[u_{h}\right]\right]\left[\left[v_{h}\right]\right] d s, \quad \forall v_{h} \in D_{k}^{\mathrm{DG}}\left(\Omega_{h}\right),
\end{aligned}
$$

with the corresponding linear form

$$
\begin{gathered}
\ell^{\mathrm{DG}}\left(v_{h}\right)=\sum_{e=1}^{N_{\mathrm{el}}} \int_{\Omega^{e}} f v_{h} d x-\sum_{\Gamma \in \Gamma_{D}} \int_{\Gamma}\left(\nabla v_{h} \cdot \mathbf{n}_{\Gamma}-\frac{\sigma}{|\Gamma|^{\beta}} v_{h}\right) L_{D} d s \\
+\sum_{\Gamma \in \Gamma_{N}} \int_{\Gamma} v_{h} L_{N} d s, \quad \forall v_{h} \in D_{k}^{\mathrm{DG}}\left(\Omega_{h}\right) .
\end{gathered}
$$

$|\Gamma|$ is defined as the two-dimensional length of the element edge, with $[[\cdot]]$ and $\{\{\cdot\}\}$ denoting the jump and average respectively across the interface between neighbouring elements $\Omega^{e_{1}}$ and $\Omega^{e_{2}}$,

$$
[[u]]=\left.u\right|_{\Omega^{e_{1}}}-\left.u\right|_{\Omega^{e_{2}}}, \quad\{\{u\}\}=\frac{1}{2}\left(\left.u\right|_{\Omega^{e_{1}}}+\left.u\right|_{\Omega^{e_{2}}}\right) .
$$

At external boundary edges we define $[[u]]=\{\{u\}\}=\left.u\right|_{\Omega^{e_{1}}}$. 
The combination of $\sigma$ and $\beta$ in Eq. (2.11) and Eq. (2.12) defines the penalty parameter and has to be chosen to be large enough to enforce coercivity of the DG-SIPG bilinear form [31]. The determination of an analytical expression for the lower bound of the penalty parameter for the DG-SIPG method to be stable and convergent was obtained in [14] for triangular elements and was revisited by the authors in [17] providing $\sigma=3 k(k+1)$ with $\beta=1$. These expressions for the penalty parameters are retained hereafter, if not stated otherwise.

\subsubsection{Matrix formulation}

Substituting the expansion (2.9) into (2.10) on a single element leads to a matrix problem to determine the global coefficients $\hat{\mathbf{u}}$. The global matrix problem is constructed by considering matrices locally on each element $\Omega^{e}$. In the $C^{0}$ formulation, ignoring contributions from boundary conditions initially, on each element we arrive at the matrix equation

$$
\left(\mathbf{L}^{e}+\alpha \mathbf{M}^{e}\right) \hat{\mathbf{u}}^{e}=\hat{\mathbf{f}}^{e},
$$

where $\mathbf{M}^{e}$ and $\mathbf{L}^{e}=\mathbf{L}_{x}^{e}+\mathbf{L}_{y}^{e}$ represent the elemental mass and Laplacian matrices defined as

$$
\left(\mathbf{M}^{e}\right)_{i j}=\int_{\Omega^{e}} \phi_{i}(\mathbf{x}) \phi_{j}(\mathbf{x}) d x, \quad\left(\mathbf{L}_{r}^{e}\right)_{i j}=\int_{\Omega^{e}} \partial_{r} \phi_{i}(\mathbf{x}) \partial_{r} \phi_{j}(\mathbf{x}) d x,
$$

for $0 \leq i, j \leq N$ where $N$ is the number of local degrees of freedom on each triangle and $\hat{\mathbf{f}}^{e}=\int_{\Omega^{e}} \phi_{i}(\mathbf{x}) f d x$. The global counterparts of the elemental matrices are constructed using the assembly matrix, so that for example $\mathbf{M}=\mathcal{A}^{\top} \mathbf{M}^{l} \mathcal{A}$ where $\mathbf{M}^{l}=\bigoplus_{e=1}^{N_{\mathrm{el}}} \mathbf{M}^{e}$ is the direct sum of the elemental mass matrices. We therefore obtain the solution $u_{h}$ by solving the matrix system

$$
\mathbf{A}^{\mathrm{CG}} \hat{\mathbf{u}}=(\mathbf{L}+\alpha \mathbf{M}) \hat{\mathbf{u}}=\hat{\mathbf{f}},
$$

where the vector $(\hat{\mathbf{f}})_{i}^{\mathrm{CG}}=\ell^{\mathrm{CG}}\left(\phi_{i}\right)$ imposes the Neumann boundary condition weakly.

In the DG formulation, we use the same definitions for the elemental mass and Laplacian matrices. Since the solution is discontinuous across elements, global matrices are easily constructed as the direct sum of local matrices (i.e. $\mathcal{A}=\mathbf{I d}$ and $\mathbf{M}=\mathbf{M}^{l}$ ). However, since information must propagate between elements, we numerically approximate the flux through common edges. In matrix form this is represented in a global sense as

$$
(\mathbf{T})_{i j}=\sum_{\Gamma \in \Gamma_{h} \cup \Gamma_{D}}\left[\frac{\sigma}{|\Gamma|^{\beta}} \int_{\Gamma}\left[\left[\phi_{i}\right]\right]\left[\left[\phi_{j}\right]\right] d s-\int_{\Gamma}\left\{\left\{\nabla \phi_{i}\right\}\right\} \cdot \mathbf{n}_{\Gamma}\left[\left[\phi_{j}\right]\right] d s-\int_{\Gamma}\left\{\left\{\nabla \phi_{j}\right\}\right\} \cdot \mathbf{n}_{\Gamma}\left[\left[\phi_{i}\right]\right] d s\right],
$$

leading to the matrix equation

$$
\mathbf{A}^{\mathrm{DG}} \hat{\mathbf{u}}=(\mathbf{L}+\alpha \mathbf{M}+\mathbf{T}) \hat{\mathbf{u}}=\hat{\mathbf{f}}
$$

with $(\hat{\mathbf{f}})_{i}^{\mathrm{DG}}=\ell^{\mathrm{DG}}\left(\phi_{i}\right)$. 


\subsubsection{Boundary conditions for the Poisson equation}

In the previous sections we omitted a description of how Dirichlet boundary conditions are imposed in the $C^{0}$ formulation. Typically, these conditions are imposed strongly by decomposing the solution $u_{h}(\mathbf{x})$ into a known solution $u_{h}^{D}(\mathbf{x})$ and an unknown homogeneous solution $u_{h}^{H}(\mathbf{x})$ so that $u_{h}(\mathbf{x})=u_{h}^{D}(\mathbf{x})+u_{h}^{H}(\mathbf{x})$ with $u_{h}^{H}\left(\Gamma_{N}\right)=0$ and $u_{h}^{D}\left(\Gamma_{D}\right)=L_{D}$. The matrix system (2.13) is then condensed so that only the submatrix corresponding to interior degrees of freedom and Neumann boundary conditions are solved for.

In order to provide a comparison with the DG-SIPG results, in which Dirichlet boundary conditions are imposed weakly, we will only consider a fully weak $C^{0}$ formulation. For the problems considered in the next section, an exact solution is known and thus we can theoretically impose boundary conditions weakly by specifying an exact Neumann condition. However, since the global Laplacian matrix has rank one fewer than the total number of degrees of freedom and is non-invertible, the solution of (2.13) cannot be determined uniquely if $\alpha=0$, as is the case for the pressure Poisson equation when equipped with only Neumann conditions. A typical solution to this is to pin a single degree of freedom to an arbitrarily chosen Dirichlet value, making the system invertible.

Since we require an entirely weak formulation, we choose instead to impose a Robin boundary condition of the form

$$
\frac{\partial u}{\partial n}+u=L_{D}
$$

In the $C^{0}$ formulation, this requires a modification of the elemental matrix $\mathbf{A}^{e}$ to sum additional contributions from a boundary mass matrix of any edges $\Gamma_{n}^{e}$ which contain a Robin condition [25]. Consequently, A becomes invertible when $\alpha=0$. Overall this is equivalent to imposing a weak Dirichlet condition through a penalty scheme and matches the strategy adopted in the DG-SIPG formulation.

\subsection{Summary of the method and algebraic formulation}

In this section we have outlined both the temporal and spatial discretisations under study in this work. We conclude by defining the full matrix formulation for the high-order splitting scheme which will be studied in the next section.

Firstly, we define two additional matrices necessary in the formulation. To calculate divergence and gradient terms, we utilise the weak derivative matrix

$$
\left(\mathbf{K}_{\mathbf{r}}^{e}\right)_{i j}=\int_{\Omega^{e}} \phi_{i}(\mathbf{x}) \partial_{r} \phi_{j}(\mathbf{x}) d \mathbf{x}
$$

for each coordinate direction $r=x, y$. Additionally, the explicitly treated non-linear terms give rise to the local matrix

$$
\mathbf{N}_{\mathbf{r}}^{e}(\mathbf{u})=\int_{\Omega^{e}} \mathbf{N}_{r}^{e}\left(\mathbf{u}^{e}\right) \phi_{i}(\mathbf{x}) d x
$$


where $\mathbf{u}^{e}$ denotes the solution evaluated at the quadrature points in element $\Omega^{e}$. The corresponding global matrices $\mathbf{K}_{\mathbf{r}}$ and $\mathbf{N}_{\mathbf{r}}(\mathbf{u})$ are constructed through the assembly matrix as seen previously. Finally, in Section 2.2.3 we considered a one dimensional elliptic problem, whilst the Navier-Stokes equations required the multidimensional version (e.g. $\mathbf{u}=(u, v)^{\top}$ in 2D). Extension to a multidimensional formulation is easily achieved by repeating the one-dimensional global matrices $\mathbf{A}^{\text {Pois }}, \mathbf{A}^{\text {Helm }}$ and $\mathbf{M}$ to the diagonal of an extended matrix and completing with zero block matrices in the off-diagonal. Then each block diagonal corresponds to an entry of the multidimensional velocity vector. Additionally, we define the 2D matrices $\mathbf{K}=\left(\mathbf{K}_{\mathbf{x}}, \mathbf{K}_{\mathbf{y}}\right)$ and $\mathbf{N}(\mathbf{u})=\left(\mathbf{N}_{\mathbf{x}}(\mathbf{u}), \mathbf{N}_{\mathbf{y}}(\mathbf{u})\right)^{\top}$ Using these matrices, we rewrite the temporal discretisation scheme in matrix form as

$$
\begin{aligned}
& \mathbf{M} \frac{\gamma_{0} \tilde{\mathbf{u}}-\alpha_{0} \hat{\mathbf{u}}^{n}-\alpha_{1} \hat{\mathbf{u}}^{n-1}}{\Delta t}=-\beta_{0} \mathbf{N}\left(\mathbf{u}^{n}\right)-\beta_{1} \mathbf{N}\left(\mathbf{u}^{n-1}\right), \\
& \mathbf{A}^{\text {Pois }} \hat{\mathbf{p}}^{n+1}=-\frac{\gamma_{0}}{\Delta t} \mathbf{K} \tilde{\hat{\mathbf{u}}}+\hat{\mathbf{f}}^{\text {Pois }}, \\
& \left(\mathbf{A}^{\mathrm{Helm}}+\frac{\gamma_{0}}{v \Delta t} \mathbf{M}\right) \hat{\mathbf{u}}^{n+1}=\frac{1}{v}\left(\frac{\gamma_{0}}{\Delta t} \mathbf{M} \tilde{\mathbf{u}}-\mathbf{K}^{\top} \hat{\mathbf{p}}^{n+1}\right)+\hat{\mathbf{f}}^{\mathrm{Helm}} .
\end{aligned}
$$

Let us note that in general, and in particular for the CG formulation, $\mathbf{A}^{\text {Pois }}$ and $\mathbf{A}^{\text {Helm }}$ denote the same matrix $\mathbf{A}$ if $\alpha=0$ (see Section 2.2.3). However, distinguishing between the matrices arising from solving either the Poisson step or the Helmholtz step, proves advantageous for the analysis and hence this notation will be maintained hereafter.

\subsection{Eigenvalue system}

To conclude the methodology section, we briefly outline the procedures used in the coming sections to calculate eigenvalues of spatial operators. In general we wish to approximate a finite subset of eigenvalues of a continuous operator whose discretisation is represented by a matrix $\mathbf{B}$. This is equivalent to defining a generalised eigenvalue system

$$
\mathbf{B} \hat{\mathbf{x}}_{i}=\lambda_{i} \mathbf{M} \hat{\mathbf{x}}_{i}
$$

where $\lambda_{i}$ denotes the $i$-th generalised eigenvalue of $\mathbf{B}, \hat{\mathbf{x}}_{i}$ is the vector of global coefficients for the $i$-th eigenvector and $\mathbf{M}$ represents the mass matrix corresponding to the spatial discretisation used to derive the matrix $\mathbf{B}$. We note that this system is equivalent to the usual eigenvalue problem $\mathbf{R} \hat{\mathbf{x}}_{i}=\lambda_{i} \hat{\mathbf{x}}_{i}$ with $\mathbf{R}=\mathbf{M}^{-1} \mathbf{B}$, as long as $\mathbf{M}$ is not singular; i.e. both CG and DG formulations provide invertible global mass matrices. In Section 4.2, the eigenvalues of various matrices are computed to derive the stability bounds of the splitting scheme. The eigenvalues included there correspond to the eigenvalues of the generalised system $\mathbf{R} \hat{\mathbf{x}}_{i}=\lambda_{i} \hat{\mathbf{x}}_{i}$. 


\section{Evidence of numerical instabilities: unsteady Stokes equations}

The time discretisation scheme defined in Section 2.1 may be used without considering the non-linear terms (i.e. $\mathbf{N}(\mathbf{u})=\mathbf{0})$ to solve the unsteady Stokes equations. In this section, the following solution for the Stokes problem $[28,32]$ is considered:

$$
\begin{aligned}
& u=e^{-\lambda t} \sin (x)(a \sin (a y)-\cos (a) \sinh (y)), \\
& v=e^{-\lambda t} \cos (x)(\cos (a y)+\cos (a) \cosh (y)), \\
& p=\lambda e^{-\lambda t} \cos (a) \cos (x) \sinh (y),
\end{aligned}
$$

with $a$ and $\lambda$ problem constants. For the first set of cases, we select $a=2.883356, \lambda=$ 9.313739 and solve this unsteady problem in a domain $\Omega=[-1,1]^{2}$ with boundary and initial conditions provided by the exact solution (but similar results are obtained when using the Neumann pressure boundary condition Eq. (2.5)). Tests are conducted using both CG and DG formulations on two meshes constituted of 72 and 128 semi-structured triangular elements (i.e. divided rectangular elements). Let us note that only equal order polynomial spaces $\left[D_{k}\left(\Omega_{h}\right)\right]_{\text {vel }}^{2}-\left[D_{k}\left(\Omega_{h}\right)\right]_{\text {press }}$ for velocity and pressure are considered. To quantify the accuracy of the methods we use the $L^{2}$ relative error norm, which is defined for velocity and pressure as:

$$
\frac{\left\|\mathbf{u}^{\text {exact }}-\mathbf{u}_{h}\right\|_{L^{2}\left(\Omega_{h}\right)}}{\left\|\mathbf{u}^{\text {exact }}\right\|_{L^{2}\left(\Omega_{h}\right)}}, \frac{\left\|p^{\text {exact }}-p_{h}\right\|_{L^{2}\left(\Omega_{h}\right)}}{\left\|p^{\text {exact }}\right\|_{L^{2}\left(\Omega_{h}\right)}},
$$

where the super-script exact denotes the analytical solution of the given variable and $\mathbf{u}_{h}$ and $p_{h}$ are the velocity and pressure obtained numerically for a given mesh.

Fig. 1 shows the $L^{2}$ relative error norm convergence for pressure and velocity obtained for time step refinement. For spatially well resolved simulations $(k \geq 7)$, convergence rates for the $L^{2}$ norm for the CG formulation are 1.98 for pressure and 2.17 for velocity. The DG formulation provides slopes of 1.82 for the pressure and 2.21 for velocity, showing that these slopes are dictated by the time advancement scheme and independent of the spatial formulation. Both continuous and discontinuous convergence rates are in very good agreement with the theoretical temporal convergence rates in the $L^{2}$ norm obtained in [20] for conformal discretisations and the splitting scheme employed, which are $\mathcal{O}\left(\triangle t^{3 / 2}\right)$ for pressure and $\mathcal{O}\left(\triangle t^{2}\right)$ for velocity.

It can be seen that for all polynomial orders $k$ the error decreases with $\triangle t$ provided that the time step remains larger that a certain $\triangle t_{\text {lim }}(h, k)$, which itself reduces as the spatial discretisation increases (using either $h$ - or $p$-refinement).

In addition to the time step refinement study, we explore the effect of varying the viscosity $v$. We fix the polynomial order to $k=4$ on a mesh comprising 128 semi-structured triangular elements and provide results obtained from both CG and DG formulations. In the Stokes problem previously defined, the kinematic viscosity is defined by the constants $a$ and $\lambda$ through the relationship $v=\lambda /\left(1+a^{2}\right)$. We therefore consider the three cases: 

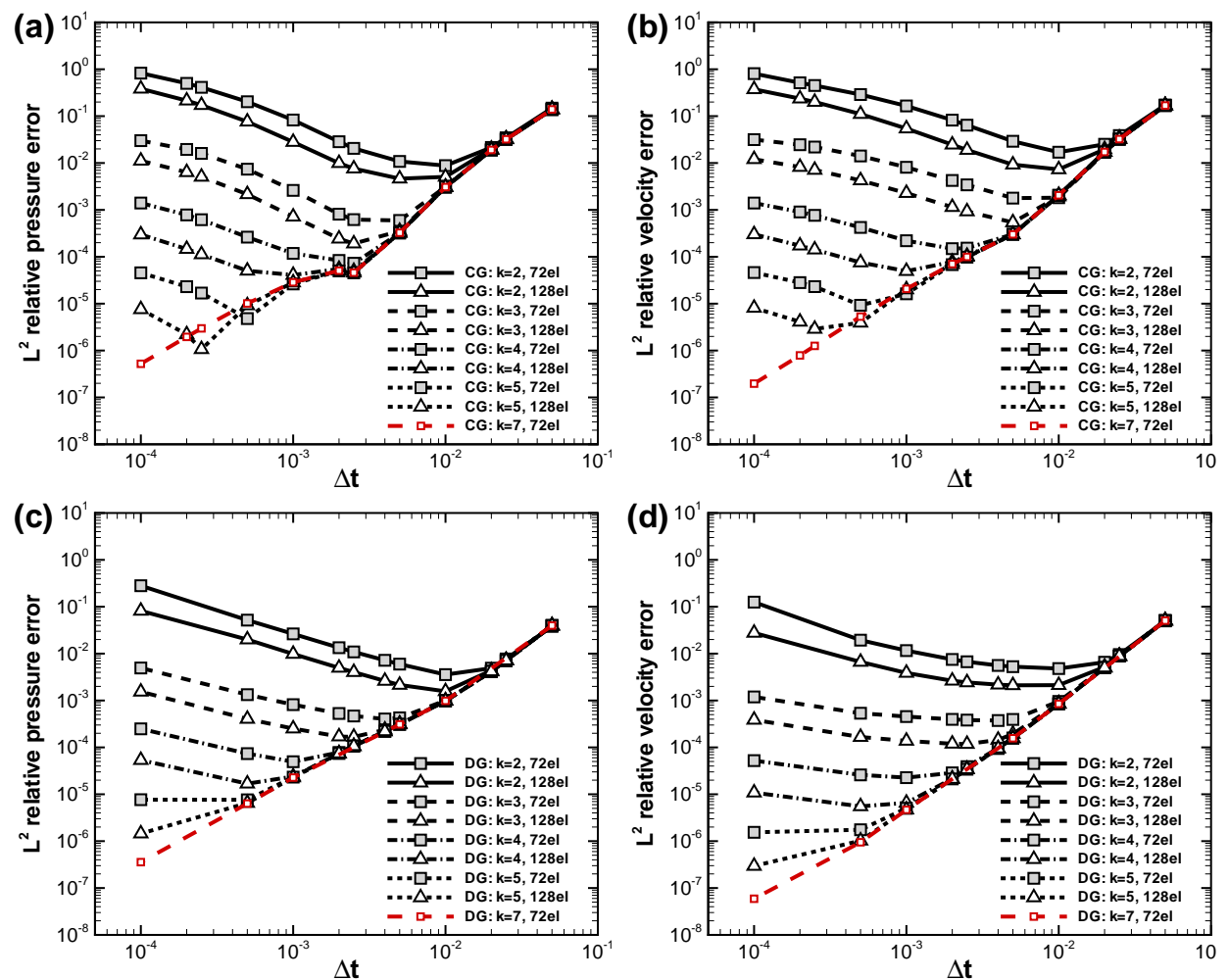

Figure 1: Time step refinement study for the unsteady Stokes equations at final time $T=0.1$, for two meshes and various polynomial orders $k . L^{2}$ relative error norm for continuous and discontinuous formulations (a) CG pressure, (b) CG velocity, (c) DG pressure and (d) DG velocity.

1. $v_{0}=1$ using $a=2.883356, \lambda=9.313739$,

2. $v_{1}=0.1$ using $a=9.598822, \lambda=9.313739$,

3. $v_{2}=0.1$ using $a=2.883356, \lambda=0.931374$,

which demonstrate how the error varies as $a$ and $\lambda$ vary independently.

Results are depicted in Fig. 2, and show that the kinematic viscosity influences the limit of stability of the scheme. In particular we observe that low viscosities require higher time steps for the calculation to remain stable. As it will be shown in following sections, the limit for stability relates to the product of the time step and the kinematic viscosity: $\kappa_{\text {lim }}=v \Delta t$, and not only to the time step.

\section{Analysis: temporal stability and the inf-sup condition}

As showed in the previous section, instabilities (or unbounded $L^{2}$ errors) may arise if for a given viscosity $v$, a small time step $\triangle t<\Delta t_{\text {lim }}$ is selected for computation. In this section, 

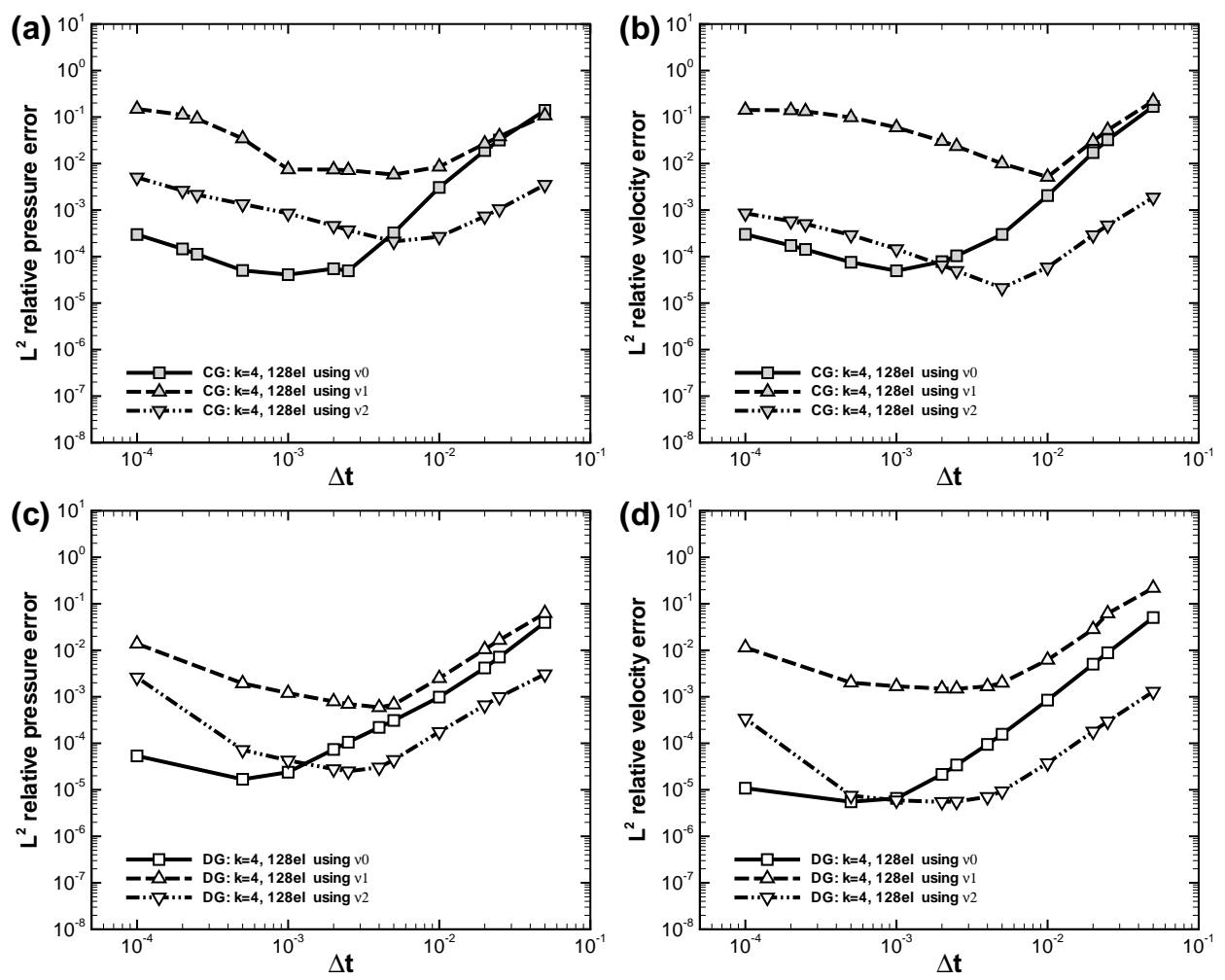

Figure 2: Time step refinement study for the unsteady Stokes equations at final time $T=0.1,128$ triangular element mesh, polynomial order $k=4$ and three kinematic viscosities $v_{0}, v_{1}$ and $v_{2}$. $L^{2}$ relative error norm for continuous and discontinuous formulations: (a) CG pressure, (b) CG velocity, (c) DG pressure and (d) DG velocity.

we explore the causes of this behaviour seeking inspiration from the work performed in [18]. In this paper, Guermond et al. provide a summary of the stability conditions for projection schemes (i.e. pressure-correction methods, velocity-correction methods and consistent splitting methods). They review different temporal schemes including the one considered in this work and address the issue: Do projection schemes require an inf-sup condition to be satisfied?

In [18], the authors consider the Chorin-Temam $[8,35]$ algorithm and detail that the stability of projection schemes can be reduced to the analysis of the equivalent steady Stokes problem. A similar approach is used in the next section to analyse the stability of the selected splitting scheme.

\subsection{Continuous system}

Using the continuous form of the NS equations, and considering the equivalent steady state problem without non-linear terms, the following matrix system for the continuous 
steady Stokes problem (i.e. the saddle point system) can be derived:

$$
\mathbf{S}=\left(\begin{array}{cc}
-v \nabla^{2} & \nabla \\
\nabla \cdot & 0
\end{array}\right)\left(\begin{array}{l}
\mathbf{u} \\
p
\end{array}\right)=\left(\begin{array}{l}
\mathbf{0} \\
0
\end{array}\right)
$$

We modify this system slightly by dividing the first equation and multiplying the second by the kinematic viscosity $v$ to obtain

$$
\mathbf{S}=\left(\begin{array}{cc}
-\nabla^{2} & \frac{1}{v} \nabla \\
v \nabla \cdot & 0
\end{array}\right)\left(\begin{array}{c}
\mathbf{u} \\
p
\end{array}\right)=\left(\begin{array}{l}
\mathbf{0} \\
0
\end{array}\right)
$$

Let us note that since the kinematic viscosity is a constant, the system defined by Eq. (4.2) is equivalent to Eq. (4.1). This less conventional form for the Stokes system is retained hereafter since it proves advantageous for the analysis of the scheme.

The spatially discretised form $\mathbf{S}_{h}$ of this Stokes system $\mathbf{S}$ must satisfy the discrete infsup (Ladyzhenskaya [26]-Babushka [3]-Brezzi [5] or LBB) condition. As introduced previously, the inf-sup condition states that an equal order discretised pressure and velocity pair do not lead a stable system. To solve this problem one can augment the velocity space with respect to the pressure space or add stabilisation terms whilst maintaining the same space dimensions for pressure and velocity.

Let us consider the semi-discrete system resulting from the splitting scheme defined in Section 2.1, where for the Stokes equations $\mathbf{N}(\mathbf{u})=\mathbf{0}$, and derive its steady state form $\left(\mathbf{u}^{n+1}=\mathbf{u}^{n}=\mathbf{u}\right.$ and $\left.p^{n+1}=p^{n}=p\right)$ using Eqs. (2.1), (2.2) and (2.3). Under the steady Stokes assumptions, the three required steps defined in Section 2.1 reduce to:

$$
\begin{aligned}
& \tilde{\mathbf{u}}=\mathbf{u}, \\
& \gamma_{0} \frac{\tilde{\mathbf{u}}-\tilde{\mathbf{u}}}{\Delta t}=-\nabla p, \\
& \gamma_{0} \frac{\mathbf{u}-\tilde{\mathbf{u}}}{\Delta t}=v \nabla^{2} \mathbf{u},
\end{aligned}
$$

where $\Delta t$ does not represent a physical time step, but is a factor that accounts for the difference between $\tilde{\tilde{\mathbf{u}}}$ and $\tilde{\mathbf{u}}$ (resp. $\mathbf{u}$ ). It is possible to eliminate the intermediate velocities $\tilde{\mathbf{u}}$ and $\tilde{\mathbf{u}}$. Firstly, let us substitute Eq. (4.3) in Eq. (4.4), take the divergence of the resulting equation and make use of $\nabla \cdot \tilde{\tilde{\mathbf{u}}}=0$; secondly, one may add Eq. (4.4) and Eq. (4.5). Finally, let us divide the first equation by $v$ and multiply the second by the same term, to obtain the following matrix form:

$$
\mathbf{S}_{\text {split }}=\left(\begin{array}{cc}
-\nabla^{2} & \frac{1}{v} \nabla \\
v \nabla \cdot & -\frac{v \triangle t}{\gamma_{0}} \nabla^{2}
\end{array}\right)\left(\begin{array}{c}
\mathbf{u} \\
p
\end{array}\right)=\left(\begin{array}{l}
\mathbf{0} \\
0
\end{array}\right) .
$$

Comparing Eq. (4.6) to Eq. (4.2), it becomes clear that the new system $\mathbf{S}_{\text {split }}$ corresponds to a pseudo-compressible scheme, where $\frac{v \Delta t}{\gamma_{0}}$ may be interpreted as the pseudocompressibility coefficient [33], or alternatively $\frac{v \Delta t}{\gamma_{0}} \nabla^{2}$ as a pressure stabilisation term 
which relaxes the incompressibility constraint (see also [30]). As noted in [18] and [19] for the original splitting scheme of Chorin and Temam, if the spatial discretisation $\left(\mathbf{S}_{\text {split }}\right)_{h}$ of the continuous system $\mathbf{S}_{\text {split }}$ uses inf-sup unstable pressure-velocity pairs, then if $\triangle t$ becomes "too small", spurious modes manifest themselves, which may lead to instabilities.

However, the present analysis and inspection of the matrix system Eq. (4.6), reveals that it is not only the time step $\Delta t$ that ensures the stability of the scheme, but the product of the time step and the kinematic viscosity $v: \kappa=v \Delta t$.

\subsection{Algebraic system}

The same approach can be used to obtain the system corresponding to the discretised algebraic scheme defined in Section 2.3. Once more, the steady Stokes problem is considered with equal order polynomial spaces for pressure and velocity. The system reads:

$$
\left(\mathbf{S}_{\text {split }}^{\text {alg }}\right)_{h}=\left(\begin{array}{cc}
\mathbf{A}^{\text {Helm }} & \frac{1}{v} \mathbf{K}^{\top} \\
v \mathbf{K} & \frac{v \Delta^{t}}{\gamma_{0}} \mathbf{A}^{\text {Pois }}
\end{array}\right)\left(\begin{array}{c}
\hat{\mathbf{u}} \\
\hat{\mathbf{p}}
\end{array}\right)=\left(\begin{array}{c}
\hat{\mathbf{f}}^{\text {Helm }} \\
v \hat{\mathbf{f}}^{\text {Pois }}
\end{array}\right) .
$$

This matrix form shows that if $v \Delta t$ becomes small, the discrete version of the saddle point Stokes system $\mathbf{S}$ (i.e. without stabilisation) is recovered, which would require an inf-sup stable pressure-velocity pair (e.g. smaller polynomial order for pressure than for velocity). It may be hypothesised that when equal order velocity/pressure pairs are used (i.e. inf-sup unstable pairs), the term $\frac{v \triangle t}{\gamma_{0}} \mathbf{A}^{\text {Pois }}$ dictates the temporal stability of the scheme. In particular, it may be intuitively argued that "enough" stabilisation is provided by the combination of:

- a large enough time step $\triangle t$,

- a high enough viscosity $v$ (resp. low enough Reynolds number Re when considering the full NS equations),

- a large enough spectral radius for the matrix $\mathbf{A}^{\text {Pois }}$ when compared to $v \Delta t$.

More precisely, one can eliminate the velocity from the system described by Eq. (4.7) to explore the solvability condition for the pressure. Assuming that $\mathbf{A}^{\mathrm{Helm}}$ is invertible (i.e. both CG and DG-SIPG method provide positive definite matrices), one obtains:

$$
\left(\frac{v \triangle t}{\gamma_{0}} \mathbf{A}^{\text {Pois }}-\mathbf{K}\left[\mathbf{A}^{\text {Helm }}\right]^{-1} \mathbf{K}^{\top}\right) \hat{\mathbf{p}}=v\left(\hat{\mathbf{f}}^{\text {Pois }}-\mathbf{K}\left[\mathbf{A}^{\text {Helm }}\right]^{-1} \hat{\mathbf{f}}^{\text {Helm }}\right) .
$$

It can be seen that the solvability for the pressure in Eq. (4.8) is determined by the invertibility of the pressure Schur complement:

$$
\operatorname{Schur}\left(\left(\mathbf{S}_{\text {split }}^{\text {alg }}\right)_{h}\right)=\frac{v \Delta t}{\gamma_{0}} \mathbf{A}^{\text {Pois }}-\mathbf{K}\left[\mathbf{A}^{\text {Helm }}\right]^{-1} \mathbf{K}^{\top},
$$


where the dependency of the discrete pressure stabilisation term $\frac{v \triangle t}{\gamma_{0}} \mathbf{A}^{\text {Pois }}$ becomes explicit.

We can provide a necessary condition for solvability by considering the $L^{2}$ norm of the Schur complement defined by Eq. (4.9):

$$
\begin{aligned}
\left\|\operatorname{Schur}\left(\left(\mathbf{S}_{\text {split }}^{\text {alg }}\right)_{h}\right)\right\|_{2} & =\left\|\frac{v \triangle t}{\gamma_{0}} \mathbf{A}^{\text {Pois }}-\mathbf{K}\left[\mathbf{A}^{\text {Helm }}\right]^{-1} \mathbf{K}^{\top}\right\|_{2} \\
& \geq C\left|\frac{v \triangle t}{\gamma_{0}}\left\|\mathbf{A}^{\text {Pois }}\right\|_{2}-\left\|\mathbf{K}\left[\mathbf{A}^{\text {Helm }}\right]^{-1} \mathbf{K}^{\top}\right\|_{2}\right|,
\end{aligned}
$$

which follows from the reverse triangle inequality, where $\|\cdot\|_{2}$ denotes the $L^{2}$ norm and $C$ is a constant independent of the discretisation. We can evaluate the worst case considering:

$$
\left\|\operatorname{Schur}\left(\left(\mathbf{S}_{\text {split }}^{\text {alg }}\right)_{h}\right)\right\|_{2} \geq C\left|\frac{v \Delta t}{\gamma_{0}} \lambda_{\max }\left(\mathbf{A}^{\text {Pois }}\right)-\lambda_{\min }\left(\mathbf{K}\left[\mathbf{A}^{\text {Helm }}\right]^{-1} \mathbf{K}^{\top}\right)\right|,
$$

where $\lambda_{\min }(\mathbf{A})$ and $\lambda_{\max }(\mathbf{A})$ are the minimum and maximum eigenvalues of a matrix $\mathbf{A}$. We further simplify notation by denoting an eigenvalue of $\mathbf{K}\left[\mathbf{A}^{\text {Helm }}\right]^{-1} \mathbf{K}^{\top}$ by $\lambda^{a}$ and an eigenvalue of $\mathbf{A}^{\text {Pois }}$ by $\lambda^{b}$.

At this point, we make use of the capability of high order CG and DG methods to perform $h$ - and $p$-refinement on the steady Stokes problem (Section 3). We calculate the eigenvalue spectrum $\lambda^{a}$ and $\lambda^{b}$ and study the effects of both types of refinement independently. We therefore see how each refinement strategy affects the stability of the temporal scheme.

For $p$-refinement, we consider semi-structured triangular meshes of 18 and 72 elements, and vary the polynomial order between $k=2$ and $k=10$, to determine how the minimum and maximum eigenvalues scale as a function of $k$. For the $h$-refinement we fix $k$ to either 2 or 10, and calculate the eigenvalues of the matrices for four triangular meshes consisting of 18, 32, 50 and 72 elements.

We find that the minimum eigenvalue $\lambda_{\min }^{a}$ for $\mathbf{K}\left[\mathbf{A}^{\mathrm{Helm}}\right]^{-1} \mathbf{K}^{\top}$ is zero, independently of $h$ and $k$ and for both CG and DG formulations, which confirms that inf-sup unstable pairs have been used.

The slope of $\lambda_{\min }^{b}$ and $\lambda_{\max }^{b}$ for $p$ - and $h$-refinement are depicted in Fig. 3(a,b) for the CG method and Fig. 3(c,d) for the DG method. As shown in Fig. 3(a) and Fig. 3(c) the variation of the spectral radius for the Poisson matrix behaves as $\lambda_{\max }^{b}(k)=\mathcal{O}\left(k^{3}\right)$ for $p$-refinement. Similarly, we find that $\lambda_{\max }^{b}(h)=\mathcal{O}\left(h^{-2}\right)$ for $h$-refinement as determined by Fig. 3(b) and Fig. 3(d). In addition, theoretical estimates (i.e. where no boundary conditions are taken into account) for these observed variations can be found in [22] for $p$-refinement, where it is derived that $\lambda_{\max }(k) \leq \mathcal{O}\left(k^{3}\right)$ and $\lambda_{\min }(k) \geq C$, where $C$ denotes a constant independent of the spatial discretisation. Similarly for $h$-refinement, theoretical results from chapter 7 of [23] determine that $\lambda_{\max }(h) \leq \mathcal{O}\left(h^{-2}\right)$ and $\lambda_{\min }(h) \geq C$ for $h$ refinement. Both of these results agree very well with the values observed in this work. 
(a)

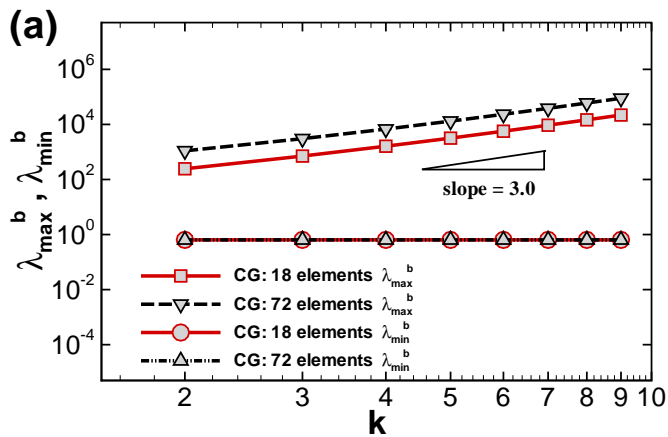

(c)

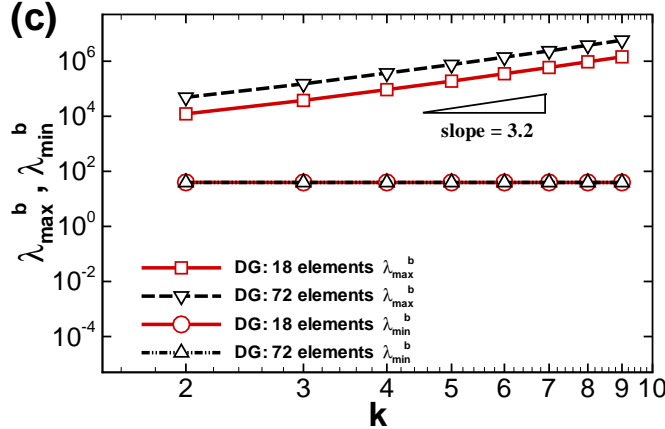

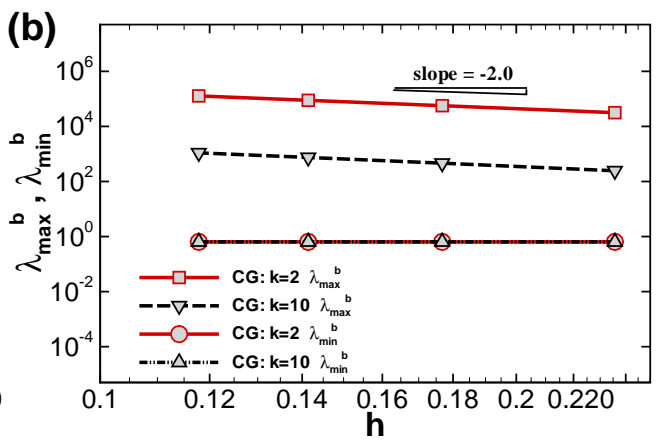

(d)

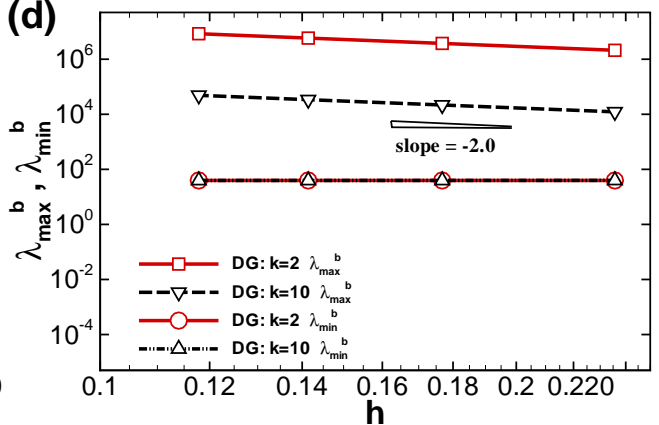

Figure 3: Eigenvalues for the Poisson matrix (Stokes problem) for continuous and discontinuous Galerkin formulations (a) CG: p-refinement, (b) CG: $h$-refinement, (c) DG: $p$-refinement and (d) DG: $h$-refinement. $k$ denotes the polynomial order and $h=\left(N_{\mathrm{el}}\right)^{-1 / 2}$ the characteristic mesh size with $N_{\mathrm{el}}$ the number of triangular elements in the mesh.

Combining these correlations, we can obtain an expression for the maximum eigenvalue as a function of mesh and polynomial refinement $\lambda_{\max }^{b}(h, k)=C_{h / p} k^{3} h^{-2}$, where $C_{h / p}$ denotes a constant independent of the spatial discretisation. Thus, the limits for solvability and associated stability outlined in Eq. (4.11) for $h$ and $p$-refinement become:

$$
\begin{aligned}
\text { general: } & \lim _{v \triangle t \rightarrow 0} \lambda^{\text {Schur }} \longrightarrow 0, \\
\text { general: } & \lim _{v \triangle t \rightarrow 0} \frac{v \Delta t}{\gamma_{0}} \lambda_{\max }{ }^{b}-\lambda_{\min }^{a} \longrightarrow 0, \\
\text { general: } & \lim _{v \triangle t \rightarrow 0} \frac{v \triangle t}{\gamma_{0}} C_{h / p} k^{3} h^{-2} \longrightarrow 0, \\
h \text {-refinement only: } & \lim _{v \triangle t \rightarrow 0} \frac{v \triangle t}{\gamma_{0}} \mathcal{O}\left(h^{-2}\right) \longrightarrow 0, \\
p \text {-refinement only: } & \lim _{v \triangle t \rightarrow 0} \frac{v \triangle t}{\gamma_{0}} \mathcal{O}\left(k^{3}\right) \longrightarrow 0 .
\end{aligned}
$$

Eqs. (4.15) and (4.16) show that if the term $\kappa=v \Delta t$ decreases faster than $\mathcal{O}\left(h^{2}\right)$ when performing $h$-refinement (e.g. low order finite element methods) or faster than $\mathcal{O}\left(k^{-3}\right)$ for $p$-refinement (e.g. high order CG or DG methods), then it is likely that the system be- 
comes ill conditioned, or equivalently requires inf-sup stable pressure-velocity pairs. We note that Guermond et al. [18] reported without explanation an observed time step limit for stability, for a similar time splitting scheme and conformal discretisations: $\triangle t \sim \mathcal{O}\left(h^{2}\right)$ for $h$-refinement and $\triangle t \sim \mathcal{O}\left(k^{-3}\right)$ for $p$-refinement, which is consistent with the present analysis. We can conclude from Eq. (4.14), that a necessary condition for the stability of $h / p$ methods, such as CG or DG, where both $h$ and $p$ refinement can be performed independently is dictated by:

$$
\kappa_{\lim }=v \triangle t \sim C_{h / p} h^{2} k^{-3},
$$

where $C_{h / p}$ denotes a constant independent of the spatial discretisation.

We also note that Maday et al. [27] showed, in the context of the Uzawa algorithm when using high order $C^{0}$ schemes, that the inf-sup constant relates to the minimum eigenvalue of the pressure Schur complement of the discrete NS system, which is consistent with our analysis.

It may be concluded that equal pressure/velocity spaces can be used in conjunction with the selected splitting scheme as long as the product of the kinematic viscosity and the time step does not becomes smaller than $\mathcal{O}\left(h^{-2} k^{3}\right)$. Therefore, high order spatial discretisations in conjunction with the selected splitting scheme may not encounter this condition, resulting in a useful method. However, low order methods (e.g. $k=1$ ) may require further stabilisation. In the following section we suggest a possible stabilisation strategy for the DG formulation. In addition, this technique proves useful to verify the previously presented analysis.

\subsection{Stabilisation for low order spatial discretisations}

The continuous Laplacian operator $\nabla^{2}$ is unique in Eq. (4.6) and when continuous discretisations are selected (e.g. CG formulation presented here). However, in the discrete case and when the DG formulation is selected, the bilinear form used for the velocity step (Helmholtz equation) and pressure step (Poisson equation) can differ. We outline this possibility by setting two distinct matrices $\mathbf{A}_{(\sigma)}^{\text {Pois }}$ and $\mathbf{A}_{(\sigma)}^{\mathrm{Helm}}$, where $\sigma$ denotes the penalty parameter for the DG-SIPG formulation. The particularity of the DG-SIPG method is that in the discrete bilinear form, the penalty parameters $\sigma$ for the pressure and the velocity are not required to be equal, leading to $\mathbf{A}_{\left(\sigma=\sigma_{P}\right)}^{\text {Pois }}$ and $\mathbf{A}_{\left(\sigma=\sigma_{H}\right)}^{\text {Helm }}$ with $\sigma_{P} \neq \sigma_{H}$.

The possibility to use two distinct Laplacian matrices provides the opportunity to verify the analysis previously introduced. In addition, we show that this particularity can be used to stabilise the method by defining a large penalty parameter for the Poisson equation (the pressure step). For example one may set:

$$
\sigma_{P}=\frac{3 k(k+1)}{|\Gamma|} \frac{1}{v \triangle t}
$$

where $\frac{3 k(k+1)}{|\Gamma|}$ is the previously derived necessary penalty parameter (see Section 2.2.2). Recalling the definition of the DG-SIPG bilinear matrix in terms of the global mass, Lapla- 


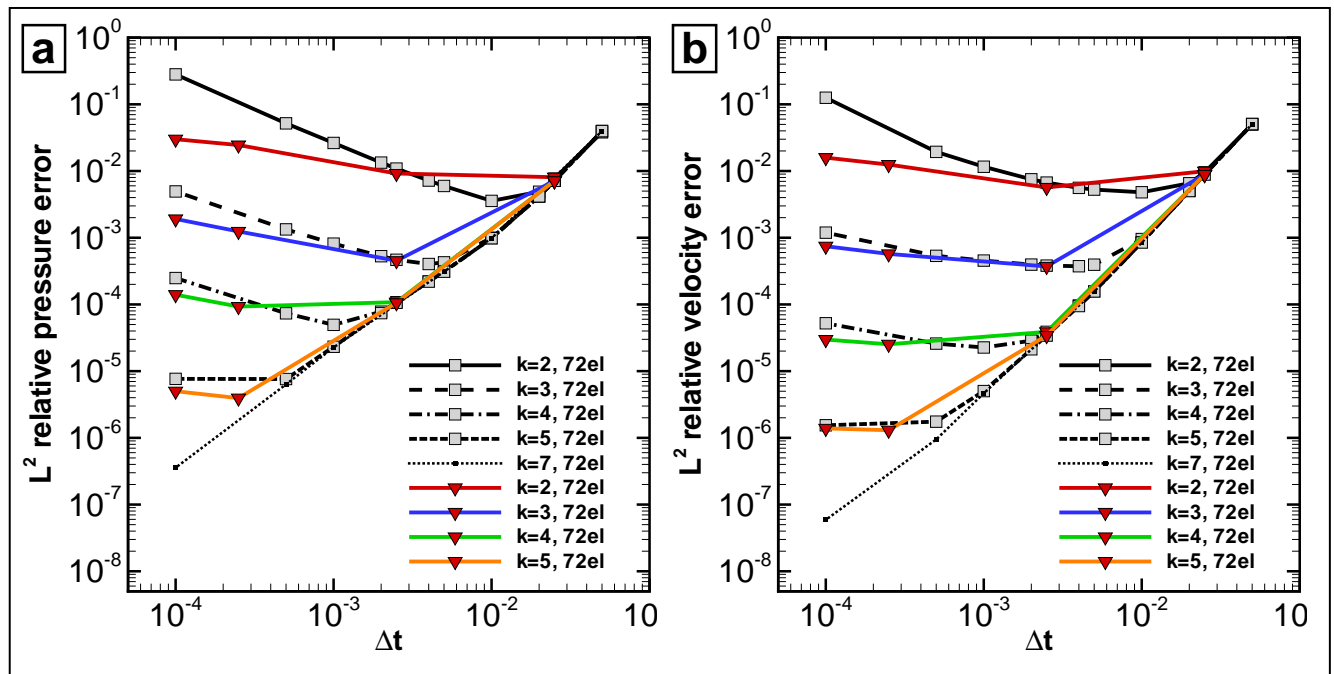

Figure 4: Time step refinement study for the unsteady Stokes equations, using the DG formulation, at final time $T=0.1$, for various polynomial orders $k . L^{2}$ relative error norm for (a) pressure and (b) velocity. Gray lines show the original scheme and colored lines the stabilised scheme.

cian and flux matrices (as introduced in Section 2.3), one can expand the Poisson bilinear matrix as:

$$
\mathbf{A}_{\left(\sigma=\sigma_{P}\right)}^{\text {Pois }}=\mathbf{L}+\alpha \mathbf{M}+\mathbf{T} \mathbf{1}+\sigma_{P} \mathbf{T} \mathbf{2},
$$

where $\mathbf{T}=\mathbf{T} \mathbf{1}+\sigma_{P} \mathbf{T} \mathbf{2}, \mathbf{T}$ is the flux matrix and:

$$
\sigma_{P}(\mathbf{T} 2)_{i j}=\left\{\frac{1}{v \triangle t} \sum_{\Gamma \in \Gamma_{h} \cup \Gamma_{D}} \frac{3 k(k+1)}{|\Gamma|} \int_{\Gamma}\left[\left[\phi_{i}\right]\right]\left[\left[\phi_{j}\right]\right] d s\right\} .
$$

Substituting Eq. (4.19) and Eq. (4.18), into the matrix form Eq. (4.7) leads:

$$
\left(\mathbf{S}_{\text {split }}^{\text {alg }}\right)_{h}=\left(\begin{array}{cc}
\mathbf{A}_{\left(\sigma_{H}\right)}^{\text {Helm }} & \frac{1}{v} \mathbf{K}^{\top} \\
v \mathbf{K} & \frac{v \triangle t}{\gamma_{0}}(\mathbf{L}+\alpha \mathbf{M}+\mathbf{T} \mathbf{1})+\frac{3 k(k+1)}{\gamma_{0}|\Gamma|} \mathbf{T} \mathbf{2}
\end{array}\right)\left(\begin{array}{c}
\hat{\mathbf{u}} \\
\hat{\mathbf{p}}
\end{array}\right)=\left(\begin{array}{c}
\hat{\mathbf{f}}_{\left(\sigma_{H}\right)}^{\mathrm{Helm}} \\
v \hat{\mathbf{f}}_{\left(\sigma_{P}\right)}^{\mathrm{Pois}}
\end{array}\right) .
$$

Inspection of this matrix system shows that for small time steps and small viscosities (resp. high Reynolds numbers when the full NS equations are considered), the term $\frac{v \triangle t}{\gamma_{0}}(\mathbf{L}+\alpha \mathbf{M}+\mathbf{T} \mathbf{1})$ tends to zero, but leaves the term $\frac{3 k(k+1)}{\gamma_{0}|\Gamma|} \mathbf{T} 2$ to provide stabilisation.

To illustrate this discussion, let us reconsider the Stokes problem and the time refinement study performed in Section 3. Fig. 4 compares the $L^{2}$ relative errors for the original scheme (gray lines shown previously in Fig. 1) and the new stabilised scheme (colored lines) for various polynomial orders using the DG formulation. As predicted, the stabilising effect for small time steps becomes noticeable and is particularly important for low order spatial discretisation. 
Effectively, this technique increases the spectral radius of the Poisson operator $\mathbf{A}_{\left(\sigma=\sigma_{P}\right)}^{\text {Pois }}$, which scales linearly with the penalty parameter: $\mathcal{O}\left(\sigma_{P}\right)$ for the Stokes problem, rendering the scheme stable for smaller values of $\kappa=v \triangle t$. In fact, this modification provides a scheme that is very similar to the stabilised technique described by Cockburn et al. in [9]. As noted in this reference, this technique requires post-processing to obtain truly divergence free velocities since the stabilisation term (i.e. the penalty term integral) alters the divergence free condition. Furthermore, this technique would increase the condition number of the pressure solve, which may pollute the solution if iterative solvers are used.

Let us note that the first author analysed in [15] the stability properties of a modified splitting scheme to compute turbulent flows (i.e. based on a Smagorinsky subgrid modelling) using the presented approach. It was shown that these type of turbulent modifications may improve the inf-sup stability enabling lower values for time steps and viscosities.

\section{Stability limit and the CFL condition}

A common practice with the splitting scheme outlined in this work is to treat the nonlinear terms explicitly, which introduces a Courant-Friedrichs-Lewy (CFL) type restriction on the maximum size of the time step. In this section we therefore explore the compatibility between the derived lower limit of stability $\kappa_{\lim }=v \Delta t$ and the CFL limit, for the full NS equations. In addition, this technique is used to verify the previous analysis.

The CFL estimate for high order spatial methods leads to $\triangle t<\Delta t_{\mathrm{CFL}} \approx h / U_{\max } k^{2}$ as shown in [25] for an advection model problem, where $h$ is the mesh element size, $U_{\text {max }}$ the maximum velocity and $k$ represents the polynomial order. The question then arises if this CFL limit, which imposes an upper bound to the time step, is in conflict with the lower bound derived in Section 4.2.

To show that the lower limit for the time step does not represent a restriction when the full Navier-Stokes equations are considered, it suffices to show that $\Delta t_{\text {lim }} \approx$ $\kappa_{\lim } / v=\left(C_{h / p} h^{2} k^{-3}\right) / \nu$ decreases faster than $\triangle t_{\mathrm{CFL}} \approx h / U_{\max } k^{2}$ as the spatial discretisation is increased, allowing for flexibility in the selection of the computational time step. This can be seen by expressing the usability condition $\Delta t_{\mathrm{CFL}}>\Delta t_{\mathrm{lim}}$ as $\triangle t_{\mathrm{CFL}}-\triangle t_{\text {lim }} \approx\left(h k^{-2} / U_{\max }-\left(C_{h / p} h^{2} k^{-3}\right) / v\right)>0$. Factoring $h k^{-2}$ leads to the condition: $\left(1 / U_{\max }-C_{h / p} h k^{-1} / v\right)>0$, which can be rearranged to:

$$
\frac{k}{h}>C_{h / p} U_{\max } / v
$$

where $C_{h / p}$ is the constant independent of the spatial discretisation and introduced in Section 4.2. This solvability condition shows that for high Reynolds number per unit length $\operatorname{Re} / L=U_{\max } / v$, based on the maximum flow velocity, it is necessary to increase the polynomial order or decrease the mesh size to obtain a useful scheme (i.e. with a 
range of time steps to select between $\Delta t_{\mathrm{CFL}}$ and $\Delta t_{\text {lim }}$ ). It may therefore be concluded that the CFL condition is compatible with the lower limit for the time step and hence the type of projections schemes can be used with pressure-velocity pairs of same degree.

\section{Conclusions}

This paper reports and analyses the limits for stability of projection type schemes when using high order pressure-velocity pairs of same degree. Two high order variational methods encompassing continuous and discontinuous Galerkin formulations have been compared showing that the limit for stability is independent of the spatial discretisation, but inherent to the temporal splitting scheme. This study has confirmed that the velocitycorrection scheme from $[24,29]$ corresponds to a stabilised scheme where the pressure stabilisation depends on the time step and the kinematic viscosity.

This work has explained the previously observed instabilities [18] for low and high order spatial discretisation when velocity or pressure correction schemes are used. Namely, it has been shown that the stability is controlled by $v \Delta t \geq C_{h / p} h^{2} k^{-3}$ leading to the conditions $v \triangle t \geq C_{p} h^{2}$ (i.e. $C_{p}=v c_{1}$ using the notation defined in the introduction) for low order finite element methods, and $v \triangle t \geq C_{h} k^{-3}$ (i.e. $C_{h}=v c_{2}$ as in the introduction) for high order spectral discretisation. These stability estimates related to the inf-sup condition of the equivalent matrix system for the splitting temporal scheme, have shown that high polynomial orders are beneficial to alleviate stability problems when selecting small time steps or small viscosities (i.e. high Reynolds numbers).

In addition, it has been shown that there is no conflict for the selection of the time step, when this condition is considered together with the Courant-Friedrichs-Lewy condition as long as high polynomials or small mesh elements are used, providing a useful nonlinear incompressible NS solver. Finally, a stabilised algorithm for low spatial resolution cases, that relies on an increased penalty parameter for the pressure step, has been used to confirm the analysis.

\section{Acknowledgments}

EF would like to thank the financial support of the John Fell Oxford University Press (OUP) fund. DM acknowledges support from the EU project IDIHOM and RW would like to thank the Research Council UK (RCUK). In addition, EF and SS would like to thank the support of the European Commission for the financial support of the ANADE project under grant contract PITN-GA-289428. Finally, we would like to kindly thank Professor Ramon Codina for the very useful discussions that helped shape this paper.

\section{References}

[1] D.N. Arnold, F. Brezzi, B. Cockburn, and L.D. Marini. Unified analysis of discontinuous Galerkin methods for elliptic problems. SIAM Journal of Numerical Analysis, 39(5):1749- 
1779, 2001.

[2] F. Auteri, J.L. Guermond, and N. Parolini. Role of the LBB condition in weak spectral projection methods: 405. Journal of Computational Physics, 174(1):405-420, 2001.

[3] I. Babuška. The finite element method with Lagrangian multipliers. Numerische Mathematik, 20(3):179-192, 1973.

[4] S. Badia and R. Codina. Algebraic pressure segregation methods for the incompressible Navier-Stokes equations. Archives of Computational Methods in Engineering, 15:1-52, 2007.

[5] F. Brezzi. On the existence, uniqueness and approximation of saddle-point problems arising from Lagrangian multipliers. ESAIM: Mathematical Modelling and Numerical Analysis, 8:129-151, 1974.

[6] F. Brezzi and K.J. Bathe. A discourse on the stability conditions for mixed finite element formulations. Computational Methods in Applied Mechanics and Engineering, 82:27-57, 1990.

[7] F. Brezzi, B. Cockburn, L.D. Marini, and E. Süli. Stabilization mechanisms in discontinuous Galerkin finite element methods. Computer Methods in Applied Mechanics and Engineering, 195(25-28):3293 - 3310, 2006.

[8] A.J. Chorin. Numerical solution of the Navier-Stokes equations. Mathematics of Computation, 22:742-762, 1968.

[9] B. Cockburn, G. Kanschat, and D. Schötzau. An equal-order DG method for the incompressible Navier-Stokes equations. Journal of Scientific Computing, 40:188-210, 2009.

[10] R. Codina. Pressure stability in fractional step finite element methods for incompressible flows. Journal of Computational Physics, 140(1):112-140, 2001.

[11] M.O. Deville, P.F. Fischer, and E.H. Mund. High-order methods for incompressible fluid flow. Cambridge monographs on applied and computational mathematics. Cambridge University Press, 2002.

[12] M.G. Duffy. Quadrature over a pyramid or cube of integrands with a singularity at a vertex. SIAM Journal on Numerical Analysis, 19(6):1260-1262, 1982.

[13] H.C. Elman, D.J. Silvester, and A.J. Wathen. Finite elements and fast iterative solvers: with applications in incompressible fluid dynamics. Oxford University Press, 2005.

[14] Y. Epshteyn and B. Rivière. Estimation of penalty parameters for symmetric interior penalty Galerkin methods. Journal of Computational and Applied Mathematics, 206:843-872, September 2007.

[15] E. Ferrer. A high order Discontinuous Galerkin - Fourier incompressible 3D Navier-Stokes solver with rotating sliding meshes for simulating cross-flow turbines. PhD thesis, University of Oxford, 2012.

[16] E. Ferrer and R. H.J. Willden. A high order discontinuous Galerkin - Fourier incompressible 3D Navier-Stokes solver with rotating sliding meshes. Journal of Computational Physics, 231(21):7037-7056, 2012.

[17] E. Ferrer and R.H.J. Willden. A high order discontinuous Galerkin finite element solver for the incompressible Navier-Stokes equations. Computers \& Fluids, 46(1):224-230, 2011.

[18] J.L. Guermond, P. Minev, and J. Shen. An overview of projection methods for incompressible flows. Computer Methods in Applied Mechanics and Engineering, 195(44-47):6011 - 6045, 2006.

[19] J.L. Guermond and L. Quartapelle. On stability and convergence of projection methods based on pressure Poisson equation. International Journal of Numerical Methods in Fluids, 26:1039-1053, 1998. 
[20] J.L. Guermond and J. Shen. Velocity-correction projection methods for incompressible flows. SIAM Journal of Numerical Analysis, 41:112-134, January 2003.

[21] R. Hartmann. Numerical analysis of higher order discontinuous Galerkin finite element methods. In H. Deconinck, editor, VKI LS 2008-08: CFD - ADIGMA course on very high order discretization methods, Oct. 13-17, 2008. Von Karman Institute for Fluid Dynamics, Rhode Saint Genèse, Belgium, 2008.

[22] Ning Hu, Xian-Zhong Guo, and I. Norman Katz. Bounds for eigenvalues and condition numbers in the p-version of the finite element method. Mathematics of Computation, 67(224):1423-1450, 1998.

[23] C. Johnson. Numerical solutions of partial differential equations by the finite element method. Cambridge University Press, 1987.

[24] G.E. Karniadakis, M. Israeli, and S.A. Orszag. High-order splitting methods for incompressible Navier-Stokes equations. Journal of Computational Physics, 97:414-443, 1991.

[25] G.E. Karniadakis and S.J. Sherwin. Spectral h/p element methods for computational fluid dynamics. Oxford Science Publications, 2005.

[26] O.A. Ladyzhenskaya. The mathematical theory of viscous incompressible flow. Gordon and Breach, New York, 2nd edition, 1969.

[27] Y. Maday, D. Meiron, A. Patera, and E. Rønquist. Analysis of iterative methods for the steady and unsteady Stokes problem: Application to spectral element discretizations. SIAM Journal on Scientific Computing, 14(2):310-337, 1993.

[28] Y. Maday, A. Patera, and E.M. Rønquist. An operator-integration-factor splitting method for time-dependent problems: Application to incompressible fluid flow. Journal of Scientific Computing, 5(4):263-292, 1990.

[29] S.A. Orszag, M. Israeli, and M. Deville. Boundary conditions for incompressible flows. Journal of Scientific Computing, 1(1):75-111, 1986.

[30] R. Rannacher. On Chorin's projection method for the incompressible Navier-Stokes equations. volume 1530 of Lecture Notes in Mathematics, pages 167-183. Springer Berlin Heidelberg, 1992.

[31] B. Rivière. Discontinuous Galerkin methods for solving elliptic and parabolic equations: theory and implementation. Society for Industrial and Applied Mathematics, Philadelphia, PA, USA, 2008.

[32] K. Shahbazi, P.F. Fischer, and C.R. Ethier. A high-order discontinuous Galerkin method for the unsteady incompressible Navier-Stokes equations. Journal of Computational Physics, 222(1):391 - 407, 2007.

[33] J. Shen. Pseudo-compressibility methods for the unsteady incompressible Navier-Stokes equations. In Guo B, editor, Proceedings of the 1994 Beijing Symposium on Nonlinear Evolution Equations and Infinite Dynamical Systems, pages 68-78. ZhongShan University Press, 1997.

[34] D.T. Steinmoeller, M. Stastna, and K.G. Lamb. A short note on the discontinuous galerkin discretization of the pressure projection operator in incompressible flow. Journal of Computational Physics, 251(0):480 - 486, 2013.

[35] A.R. Temam. Une méthode d'approximation de la solution des équations de Navier-Stokes. Bulletin de la Société Mathématique de France, 96:115-152, 1968. 